\title{
INFLUÊNCIA DA COMPACTAÇÃO DO SOLO NA PRODUTIVIDADE DA REBROTA DE EUCALIPTO ${ }^{1}$
}

\author{
Renato Antonio Dedecek² e José Luiz Gava ${ }^{3}$
}

\begin{abstract}
RESUMO - Normalmente, as áreas de rebrota sofrem a influência das operações de colheita, aplicação de herbicidas, adubos e resíduos, que concorrem para a compactação do solo. Em solos de texturas diferentes em áreas da Cia. Suzano de Papel e Celulose, nos municípios de Itatinga, SP, e São Miguel Arcanjo, SP, foram selecionadas 30 linhas de plantio para determinação da produtividade de eucalipto (Eucalyptus grandis Hill ex Maiden) aos sete anos de idade, bem como atributos físico-hídricos do solo a $50 \mathrm{~cm}$ da linha de plantio. Foram obtidas amostras indeformadas de solo em três profundidades: 0 a 10, 10 a 20 e 20 a $30 \mathrm{~cm}$; a resistência do solo foi obtida com penetrômetro - SC 60 - até $60 \mathrm{~cm}$ de profundidade. As linhas de eucaliptos foram separadas, segundo a intensidade de movimentação das máquinas de colheita, em corte, galhada e tráfego. A redução da produtividade do eucalipto chegou até dois terços, comparando-se linhas de tráfego intenso das máquinas e sem tráfego. A camada superficial do solo $(0 \mathrm{a} 10 \mathrm{~cm})$ recuperou parcialmente a estrutura durante os sete anos. A compactação foi máxima na camada de 10 a $20 \mathrm{~cm}$, no solo argiloso e na camada de 20 a $30 \mathrm{~cm}$, no solo arenoso. Houve correlação negativa $\left(\mathrm{r}^{2}=0,86\right)$ entre a densidade global, na profundidade de 10 a $20 \mathrm{~cm}$, e o volume de madeira das linhas de eucalipto no solo arenoso. No solo argiloso, essa correlação $\left(\mathrm{r}^{2}=0,77\right)$ negativa ocorreu na profundidade de 20 a $30 \mathrm{~cm}$.
\end{abstract}

Palavras-chave: Atributos físicos do solo, colheita de eucalipto e resistência do solo.

\section{INFLUENCE OF SOIL COMPACTION ON EUCALYPTUS COPPICE PRODUCTIVITY}

\begin{abstract}
In areas of eucalyptus regrowth, field operations such as harvesting, pesticides spraying, fertilization and manuring contribute to soil compaction. In two areas of eucalypt (Eucalyptus grandis Hill ex Maiden) regrowth, with soil of different textures, belonging to Cia. Suzano of Paper and Cellulose, thirty planting rows were selected to evaluate eucalyptus productivity at seven years of age and soil physical characteristics. Undisturbed soil samples were obtained at three depths: 0 - 10; 10 - 20 and 20 - 30 cm; and soil resistance at $60 \mathrm{~cm}$, using a penetrometer $S C-60$. Negative correlation between soil density and eucalypt yield was obtained at $10-20 \mathrm{~cm}\left(r^{2}=0,86\right)$ depth in sandy soil and, at $20-30 \mathrm{~cm}$ in clayey soil $\left(r^{2}=0,77\right)$. At same depths and textures, correlation coefficients between soil penetrometer resistance and eucalyptus productivity were 0,29 and 0,93, respectively. Correlation coefficients between soil bulk density and soil penetrometer resistance were 0,84, in sandy soil and 0,68 in clayey soil. In traffic lanes, eucalyptus productivity was reduced up to $66 \%$ compared to non-traffic lanes due to soil compaction. Soil surface layer seems to recover its original soil bulk density values seven years after harvesting, and soil compaction reached its maximum at $10-20$ $\mathrm{cm}$ depth on clayey soil and, $20-30 \mathrm{~cm}$ on sandy soil. There was negative correlation $\left(r^{2}=0,86\right)$ between soil bulk density (10-20 cm layer), and eucalyptus wood volume on sandy soil. On clayey soil, this negative correlation $\left(r^{2}=0,77\right)$ occurred at $20-30 \mathrm{~cm}$ depth.
\end{abstract}

Key words: soil physical characteristics, eucalypt harvesting, soil penetrometer resistance.

\footnotetext{
${ }^{1}$ Recebido em $1^{\circ} .04 .2003$ e aceito para publicação em 20.04.2005.

${ }^{2}$ EMBRAPA/Florestas, Caixa Postal 319, 83411-000 Colombo-PR. E-mail: <dedecek@ cnpf.embrapa.br>.

${ }^{3}$ Setor de Manejo, Solos e Nutrição da Cia. Suzano de Papel e Celulose, 18200-000 Itapetininga-SP.
} 


\section{INTRODUÇÃO}

A colheita mecanizada de florestas envolve tráfego intenso e pesado de máquinas sobre o solo e tem sido a principal causa da compactação dos solos. $\mathrm{O}$ impacto mais significativo nos atributos físicos do solo sob florestas ocorre em associação com operações de colheita, baldeio e subseqüente preparo do solo para rebrota ou plantio do ciclo seguinte. Essas atividades alteram a estrutura e os atributos físico-hídricos do solo, dificultando o crescimento e a distribuição das raízes no solo e, consequientemente, o desenvolvimento das florestas. Misra e Gibbons (1996) reportaram a redução no comprimento das raízes primárias e laterais de eucalipto em 71 e $31 \%$, respectivamente, com um aumento na resistência do solo à penetração de 0,4 a 4,2 MPa. Com o aumento da densidade global devido à colheita, efetuada 23 anos antes, a redução da altura total, do diâmetro e do volume de Pinus ponderosa, foi de 5, 8 e $20 \%$, respectivamente (FROELICH et al., 1986). Para Eucalyptus regnans, Rab (1996) encontrou reduções no peso de raízes de $18 \%$, quando a densidade global passou de 0,7 a $0,9 \mathrm{Mg} \cdot \mathrm{m}^{-3}$.

Segundo Matthies et al. (1995), qualquer solo sofre mudanças mensuráveis na estrutura após a passagem de um veículo se a carga exceder sua estabilidade inerente, que, de forma geral, situa-se ao redor de $50 \mathrm{KPa}$. Dependendo da relação carga/distribuição nas rodas dos veículos, a compactação de um solo pode chegar até $80 \mathrm{~cm}$ de profundidade, ressaltando-se que a compactação máxima situa-se na profundidade entre 30 e $55 \mathrm{~cm}$ (WARKOTSCH et al., 1994). Após 27 passadas de uma máquina em solo anteriormente bem-estruturado, a profundidade do sulco feito pelo pneu chegou a 10 cm, e a resistência do solo à penetração aumentou duas a três vezes, segundo Jakobsen e Greacen (1985).

Huang et al. (1996) afirmaram que a compactação do solo é o resultado da redução da macroporosidade, principalmente dos poros maiores que $3 \mathrm{~mm}$ de diâmetro. Para Lacey et al. (1994), as mudanças nos atributos físicos do solo após a colheita de florestas foram no sentido de aumentar a densidade e a resistência do solo e de reduzir a permeabilidade do solo saturado. A densidade global média, na camada de 0 a $5 \mathrm{~cm}$, após a passagem da cortadeira e do autocarregável foi de $0,80 \mathrm{Mg} \cdot \mathrm{m}^{-3}$ na linha de tráfego, $0,77 \mathrm{Mg} \cdot \mathrm{m}^{-3}$ nas áreas de corte e $0,42 \mathrm{Mg} \cdot \mathrm{m}^{-3}$ nas áreas sem tráfego (SHETRON et al., 1988).

R. Árvore, Viçosa-MG, v.29, n.3, p.383-390, 2005
Para Theodorou et al. (1991), em solo com capacidade de aeração menor que $10 \%$ houve retardamento no crescimento de raízes. Macroporos, com mais de $6 \mathrm{~mm}$ de diâmetro, apresentaram as seguintes proporções após a colheita, segundo Incerti et al. (1987): $28,6 \%$ em área sem tráfego, $19,8 \%$ na área de corte, $8,2 \%$ na linha de armazenamento dos troncos cortados e 9,7\% na linha de tráfego. Essas duas últimas áreas, com espaço aéreo menor que $10 \%$, representam aproximadamente $10 \%$ da área total de uma floresta após a colheita.

Este trabalho teve como objetivo avaliar as modificações de alguns atributos físicos do solo decorrentes das operações de colheita mecanizada com a produtividade da rebrota de eucalipto, em dois tipos de solos.

\section{MATERIAL E MÉTODOS}

Em áreas de rebrota de um plantio comercial de Eucalyptus grandis da Cia., Suzano de Papel e Celulose foram selecionados dois talhões, com florestas de sete anos de idade: o primeiro no Município de São Miguel Arcanjo-SP (S 2351' e W 47 ${ }^{\circ} 54^{\prime}$ ), em solo classificado como Latossolo Vermelho A moderado textura argilosa, relevo suave-ondulado (solo argiloso) e o segundo em Itatinga, SP (S2315' e W47 $51^{\circ}$ '), em Neossolo Quartzarênico A moderado, textura arenosa (solo arenoso).

No Quadro 1 estão reportados os dados de algumas características químicas e granulométricas dos dois solos que constam deste estudo, obtidos em amostras coletadas a $50 \mathrm{~cm}$ da linha de plantio nas profundidades de 0 a $10 \mathrm{~cm}, 10$ a $20 \mathrm{~cm}$ e 20 a $30 \mathrm{~cm}$. Nesses mesmos locais e profundidades foram coletadas amostras de solo indeformadas pelo uso de anéis volumétricos com capacidade para $100 \mathrm{~cm}^{3}$, para determinação de: densidade global (BLACK, 1965), macro (tensão de $-6 \mathrm{kPa}$ ), micro e porosidade total (OLIVEIRA, 1968), condutividade hidráulica saturada (KLUTE e DIRKSEN, 1986) e retenção de água nas tensões de -6, -10, -100 e - $1.500 \mathrm{kPa}$ (BLACK, 1965), visando constituir a curva de retenção de água. Os valores de água disponível referem-se aos teores de umidade volumétrica entre as tensões de -10 e - $1.500 \mathrm{kPa}$. As análises de fertilidade e granulometria (VETTORI, 1969) e dos atributos físicos do solo foram efetuadas segundo a metodologia descrita em Embrapa (1997). As médias apresentadas nas propriedades físicas são resultados de três repetições. 
Quadro 1 - Alguns atributos químicos de dois solos de textura diferente, por profundidade Table 1 -Some chemical properties of two soils of different textures, per depth

\begin{tabular}{|c|c|c|c|c|c|c|c|c|c|c|}
\hline \multirow[t]{2}{*}{ Profundidade } & \multirow[t]{2}{*}{$\mathrm{pH}$} & \multirow{2}{*}{$\begin{array}{l}\text { Carbono } \\
\text { Orgânico }\end{array}$} & \multirow[t]{2}{*}{ CTC } & \multicolumn{2}{|c|}{ Saturação } & \multirow[t]{2}{*}{$\mathrm{P}$} & \multicolumn{2}{|c|}{ AREIA } & \multirow[t]{2}{*}{ Silte } & \multirow[t]{2}{*}{ Argila } \\
\hline & & & & Bases & $\mathrm{Al}$ & & Fina & Grossa & & \\
\hline $\mathrm{cm}$ & & $\mathrm{g} / \mathrm{kg}$ & $\mathrm{cmol}_{\mathrm{c}} / \mathrm{dm}^{3}$ & \multicolumn{2}{|c|}{$\%$} & $\mathrm{mg} / \mathrm{dm}^{3}$ & \multicolumn{4}{|c|}{$\mathrm{g} / \mathrm{kg}$} \\
\hline \multicolumn{11}{|c|}{ SOLO ARENOSO } \\
\hline 0 a 10 & 4,40 & 8,2 & 5,9 & 2,7 & 83 & 1,1 & 440 & 470 & 10 & 80 \\
\hline 10 a 20 & 4,48 & 7,4 & 5,1 & 1,9 & 88 & 0,9 & & & & \\
\hline 20 a 30 & 4,66 & 6,5 & 4,9 & 2,3 & 85 & 1,3 & & & & \\
\hline \multicolumn{11}{|c|}{ SOLO ARGILOSO } \\
\hline 0 a 10 & 4,83 & 29,5 & 13,4 & 7,6 & 56 & 0,3 & 100 & 120 & 190 & 590 \\
\hline 10 a 20 & 4,78 & 26,1 & 11,9 & 4,1 & 73 & 0,1 & & & & \\
\hline 20 a 30 & 4,83 & 20,3 & 10,0 & 3,2 & 79 & 0,5 & & & & \\
\hline
\end{tabular}

A colheita nestas áreas compreende o uso da motosserra para corte, sendo o baldeio feito com caminhão com pneus de alta pressão (caminhão-toco). O espaçamento, nos dois locais, foi de $3 \mathrm{~m}$ entre linhas de plantio e $1 \mathrm{~m}$ e meio entre plantas na linha, e a colheita foi efetuada em 1989. As amostragens reportadas neste trabalho foram efetuadas em dezembro de 1996, quando as rebrotas estavam completando sete anos, após a primeira colheita.

Em cada solo foram demarcadas três parcelas contíguas. Cada parcela era composta de cinco linhas e 20 plantas por linha, ocorrendo em cada parcela: a) duas linhas sem depósito de madeira e sem tráfego de máquina (corte), b) uma linha com depósito de madeira sem tráfego (galhada) e c) duas linhas de retirada de madeira e de tráfego (tráfego).

Em cada parcela foram medidas altura e DAP (diâmetro à altura do peito - 1,30 m) de 20 árvores em cada linha e a resistência do solo com penetrógrafo - Soil Control 60, até a profundidade de $60 \mathrm{~cm}$. A umidade do solo por ocasião da amostragem se encontrava nos dois solos, a uma tensão de aproximadamente $-300 \mathrm{kPa}$.

\section{RESULTADOS E DISCUSSÃO}

Os dados de produtividade de eucalipto nos dois locais, de acordo com as três classes de linhas de colheita, são apresentados no Quadro 2. Houve diferença significativa de volume de madeira entre as três classes de linha de colheita, com diferenças de até dois terços a menos nas linhas com tráfego. Rab (1994) reportou que reduções de mais de $50 \%$ no incremento de altura e diâmetro de Eucalyptus regnans foram observadas em densidades de 0,91 e $0,96 \mathrm{Mg} \cdot \mathrm{m}^{-3}$ e que esses incrementos cessariam em solos com densidades de
1,32 a 1,43 Mg.m ${ }^{-3}$, respectivamente. Notou-se um comportamento diferenciado, de altura e DAP, com relação ao volume de madeira, para o solo arenoso. Enquanto altura e DAP para as linhas de galhada e corte não diferiram entre si, para volume de madeira houve diferença significativa nas três classes de intensidade de tráfego e manejo de resíduos. Segundo Rab (1994), o crescimento em altura e diâmetro de $E$. regnans com um ano de idade correlacionou-se negativamente com a densidade global na camada de 0 a $10 \mathrm{~cm}$, tendo maior efeito na altura que no diâmetro. No solo argiloso deste trabalho, a amplitude de variação de produtividade entre as diferentes linhas de tráfego foi menor e o comportamento, idêntico para todos os parâmetros considerados: altura, DAP e volume.

Observa-se, no Quadro 3, que os valores de densidade global do solo argiloso são bem menores do que os daquele de textura arenosa. No entanto, a amplitude de variação entre a camada mais e a menos

Quadro 2 - Produtividade da rebrota de eucalipto em solos de textura diferente, por linha de intensidades de tráfego na colheita mecanizada

Table 2 - Eucalyptus coppice productivity in soil of different textures, per traffic intensity lane

\begin{tabular}{lcccc}
\hline Local & Linha & Altura & DAP & Volume \\
\cline { 2 - 5 } & & $\mathrm{m}$ & $\mathrm{cm}$ & $\mathrm{m}^{3}$ \\
\hline Solo arenoso & Galhada & $17,1 \mathrm{a}^{1}$ & $11,9 \mathrm{a}$ & $531,7 \mathrm{a}$ \\
& Corte & $16,0 \mathrm{a}$ & $10,7 \mathrm{a}$ & $350,1 \mathrm{~b}$ \\
& Tráfego & $14,0 \mathrm{~b}$ & $8,7 \mathrm{~b}$ & $221,4 \mathrm{c}$ \\
\hline Solo argiloso & Galhada & $20,7 \mathrm{a}$ & $13,1 \mathrm{a}$ & $734,7 \mathrm{a}$ \\
& Corte & $19,6 \mathrm{ab}$ & $11,9 \mathrm{ab}$ & $605,3 \mathrm{ab}$ \\
& Tráfego & $18,8 \mathrm{~b}$ & $11,3 \mathrm{~b}$ & $489,3 \mathrm{~b}$ \\
\hline
\end{tabular}

${ }^{1}$ Médias seguidas por uma mesma letra na coluna e dentro do mesmo solo não diferem entre si, estatisticamente, pelo teste de Tukey a $5 \%$. 
compactada nos dois locais é muito próxima - 0,26 $\mathrm{Mg} . \mathrm{m}^{-3} \mathrm{em}$ solo argiloso e $0,31 \mathrm{Mg} \cdot \mathrm{m}^{-3} \mathrm{em}$ solo arenoso. O coeficiente de variação nas análises realizadas foi sempre da ordem de 5 a $7 \%$, muito baixo, o que permitiu que pequenas diferenças fossem significativas. Podese afirmar que há uma compactação do solo causada pelo trânsito de equipamentos na colheita da floresta e que esta alteração se aprofunda até no máximo 30 $\mathrm{cm}$ no solo arenoso e $20 \mathrm{~cm}$ no argiloso nas condições do presente estudo. Fernandes (1998), avaliando o efeito de máquinas usadas na colheita florestal, em Latossolo Vermelho textura argilosa, encontrou os maiores valores $\left(0,97\right.$ a $\left.1,09 \mathrm{Mg} \cdot \mathrm{m}^{-3}\right)$ na profundidade de 15 a $30 \mathrm{~cm}$.

Conforme se observa no Quadro 3, não há diferença significativa entre as densidades do solo das três classes de linhas de colheita, na profundidade de 0 a $10 \mathrm{~cm}$, nos dois solos. Três hipóteses podem ser levantadas: a). o processo de secagem/umedecimento do solo auxilia a recuperação da sua estrutura, mais rapidamente quanto mais freqüente forem essas alternâncias; b). o teor de matéria orgânica sempre é maior na superfície do solo, e durante os sete anos de cultivo do eucalipto muito material orgânico foi adicionado ao solo; e c). a obtenção de amostras indeformadas representativas na superfície do solo é sempre mais difícil, principalmente quando se trata de solo arenoso. Mas isso não deixa de ser surpreendente, uma vez que foi a camada de solo exposta diretamente ao contato com as máquinas de colheita utilizadas. No entanto, nas camadas subsuperficiais as alternâncias no teor de umidade do solo são menos freqüentes e menos intensas, como também é menor o teor de matéria orgânica no solo, para que permita a recuperação de sua estrutura.

Os valores de resistência do solo à penetração (Figuras 1 e 2) indicam uma maior semelhança entre os tratamentos na camada superficial do solo, principalmente no solo de textura arenosa (Figura 2). Também, o ponto de máxima compactação do solo ocorre em profundidades distintas nos dois locais, enquanto no solo argiloso se situa na camada de 10 a $20 \mathrm{~cm} \mathrm{e}$ no arenoso, na de 20 a $30 \mathrm{~cm}$ (Figura 1), concordando também com os valores de densidade global mostrados na Tabela 3. Segundo Sands et al. (1991), em solos de textura média densidades acima de $1,35 \mathrm{Mg} \cdot \mathrm{m}^{-3}$ diminuem o crescimento do caule e das raízes de Pinus radiata; e em solos com resistência ao penetrômetro acima de 3 Mpa ocorre severa restrição ao crescimento das raízes.

Observando as Figuras 1 e 2, pode-se concluir que o penetrógrafo usado não foi sensível o suficiente para avaliar a diferença entre os tratamentos "linha de corte" e "de galhada". Também nesse caso, os valores são maiores em solo arenoso (Figura 2) e a amplitude de variação dos valores de resistência do solo, maior neste. Estatisticamente, no nível de 5\% de significância a densidade global nas linhas de corte e galhada não diferiu, principalmente em solo argiloso (Quadro 3).

Quadro 3 - Densidade global do solo $\left(\mathrm{Mg} / \mathrm{m}^{3}\right)$ a $50 \mathrm{~cm}$ da linha de plantio de eucalipto, de acordo com a intensidade de tráfego na colheita, em três profundidades e solos de textura diferente

Table 3 - Soil bulk density at $50 \mathrm{~cm}$ from eucalyptus planting row, according to traffic intensity lane, at different soil depths and for different soil textures

\begin{tabular}{|c|c|c|c|c|c|}
\hline \multirow{2}{*}{$\frac{\text { PROF. }}{\text { LINHA }}$} & \multirow[t]{2}{*}{0 a $10 \mathrm{~cm}$} & \multicolumn{2}{|c|}{10 a $20 \mathrm{~cm}$} & \multicolumn{2}{|c|}{20 a $30 \mathrm{~cm}$} \\
\hline & & LINHA & & LINHA & \\
\hline & \multicolumn{5}{|c|}{ SOLO ARENOSO } \\
\hline Tráfego & $1,346 \mathrm{a}$ & Tráfego & $1,601 \mathrm{a}$ & Tráfego & $1,666 \mathrm{a}$ \\
\hline Corte & $1,385 \mathrm{a}$ & Corte & $1,584 \mathrm{ab}$ & Corte & $1,598 \mathrm{ab}$ \\
\hline Galhada & $1,352 \mathrm{a}$ & Galhada & $1,495 b$ & Galhada & $1,553 \mathrm{~b}$ \\
\hline & \multicolumn{5}{|c|}{ SOLOARGILOSO } \\
\hline Tráfego & $1,125 \mathrm{a}$ & Tráfego & $1,280 \mathrm{a}$ & Tráfego & $1,215 \mathrm{a}$ \\
\hline Corte & $1,104 \mathrm{a}$ & Corte & $1,106 \mathrm{~b}$ & Corte & $1,019 \mathrm{~b}$ \\
\hline Galhada & $1,068 \mathrm{a}$ & Galhada & $1,095 \mathrm{~b}$ & Galhada & $1,110 \mathrm{~b}$ \\
\hline
\end{tabular}

${ }^{1}$ Médias seguidas de uma mesma letra na coluna e dentro do mesmo solo não diferem entre si, estatisticamente, pelo teste de Tukey a $5 \%$.

R. Árvore, Viçosa-MG, v.29, n.3, p.383-390, 2005 


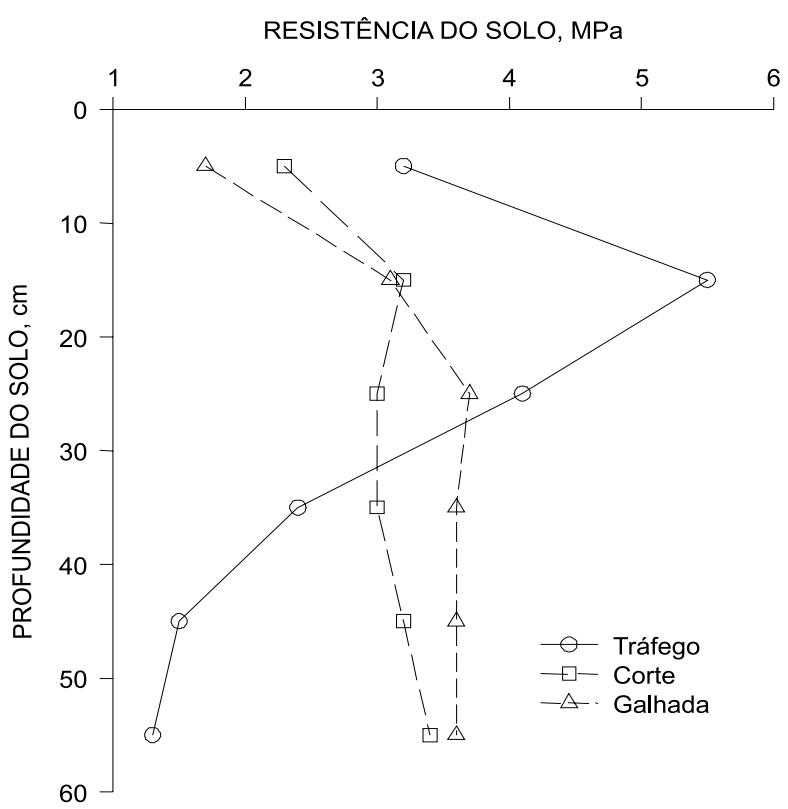

Figura 1 - Distribuição da resistência do solo à penetração em profundidade, por linha de intensidade de tráfego em solo argiloso.

Figure 1 -Distribution of soil resistance to penetrometer per soil depth and traffic intensity lane, in clayey soil.

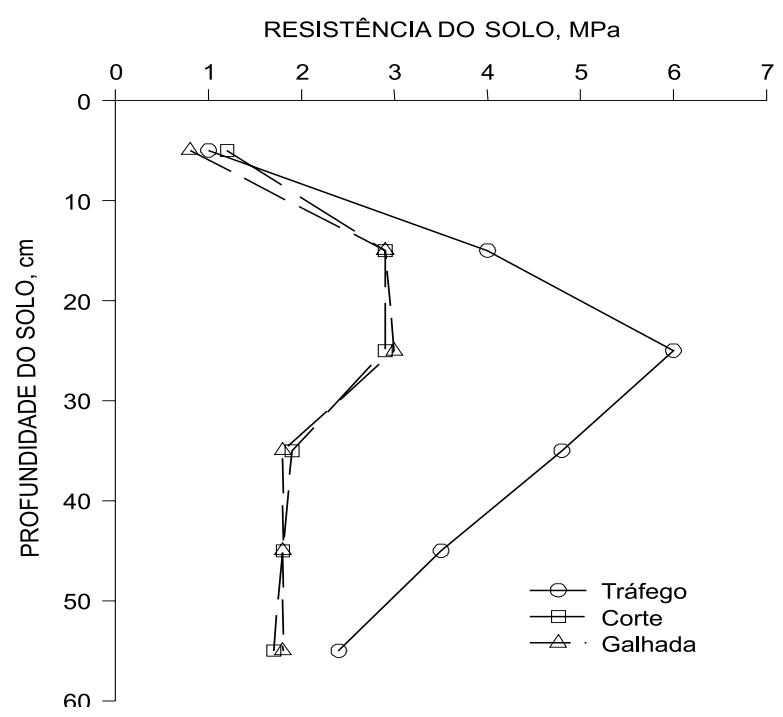

Figura 2 - Distribuição da resistência do solo à penetração em profundidade, por linha de intensidade de tráfego, em solo arenoso.

Figure 2 -Distribution of soil resistance to penetrometer per soil depth and traffic intensity lane, in sandy soil.
Com o intuito de usar um número maior de dados para um estudo de regressão linear entre as variáveis: densidade global, resistência do solo ao penetrômetro e produção em volume de madeira, essas comparações foram feitas para cinco linhas, sem agrupá-las de acordo com o tráfego de máquinas de colheita. Houve boa correlação entre a densidade global e a resistência do solo à penetração (Quadro 4). No entanto, na correlação entre o volume de madeira da rebrota de eucalipto medido aos sete anos e a densidade global, observaram-se valores de $r^{2}$ superiores, principalmente na camada de 10 a $20 \mathrm{~cm}$ de profundidade do solo. Vereficou-se que a avaliação da compactação do solo com o penetrômetro indicou maiores diferenças entre os tratamentos na superfície do solo. Notam-se, na Figura 1, valores muito baixos de resistência do solo na linha de tráfego nas maiores profundidades, o que talvez seja em decorrência do grande esforço necessário para romper a camada de 10 a $20 \mathrm{~cm}$. Segundo Voorhees et al. (1989), a resistência do solo ao penetrômetro é um indicador mais sensível aos planos de fratura ou de fraqueza do solo e a densidade global, indicativa de alterações no volume de poros. No caso dos solos em estudo, talvez estes estejam mais sujeitos a alterações na estrutura do que propriamente na formação de camadas de impedimento. Comumente os solos arenosos possuem elevada macroporosidade, sua compactação tem que ser extremamente alta para haver redução drástica dessa porosidade. Já os solos argilosos, principalmente quando com umidade acima da capacidade de campo, tendem a ser compactados facilmente, diminuindo sua porosidade e podendo formar camadas compactadas.

Inversamente aos dados de densidade e resistência do solo, a condutividade saturada deste diminuiu nas camadas de 10 a $20 \mathrm{~cm}$ no solo argiloso e de 20 a 30 cm em solo arenoso (Quadro 5), na linha de tráfego, corroborando os achados de Lacey et al. (1994). É interessante notar que, no solo arenoso, o valor da condutividade hidráulica saturada da camada adensada $(20$ a $30 \mathrm{~cm})$ é superior a qualquer valor dessa característica no solo argiloso. Pode-se observar que, tanto para macroposidade quanto para microporosidade e volume de água disponível (entre -6 e -1.500 KPa), os valores nas camadas compactadas, nos solos arenoso e argiloso, são menores em relação à linha considerada (tráfego, corte e galhada), mas não são valores absolutamente menores. Segundo Incerti et al. (1987), os macroporos

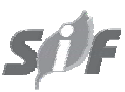

R. Árvore, Viçosa-MG, v.29, n.3, p.383-390, 2005 
maiores que 0,06 mm de diâmetro são responsáveis pela aeração do solo. Como mostrado no Quadro 5, os macroporos nos dois solos apresentaram-se com porcentagem superior a $10 \%$, o que leva a supor que não é essa a característica responsável pela redução no crescimento dos eucaliptos. Quando a intensidade de compactação não é muito grande, a quantidade de poros de tamanho intermediário pode ser aumentada e os poros internos dos agregados, permanecer inalterados.

Quadro 4 - Coeficiente de correlação $\left(\mathrm{r}^{2}\right)$ entre as variáveis resistência do solo, densidade global e volume de madeira, altura e DAP das árvores, usando-se os valores obtidos em cinco linhas de colheita, em dois solos e três profundidades

Table 4-Correlation coefficient $\left(r^{2}\right)$ among soil resistance, bulk density and wood volume, tree height and DBH, using values obtained from five harvesting rows, two soils and three depths

\begin{tabular}{|c|c|c|c|c|c|}
\hline Profundidade & Variável & Densidade Global & Volume de Madeira & Altura & DAP \\
\hline $\mathrm{cm}$ & & \multicolumn{4}{|c|}{ Solo Arenoso } \\
\hline Todas & Resistência & 0,84 & & & \\
\hline \multirow[t]{2}{*}{0 a 10} & Resistência & & 0,23 & 0,18 & 0,25 \\
\hline & Densidade & & 0,07 & 0,01 & 0,01 \\
\hline \multirow[t]{2}{*}{10 a 20} & Resistência & & 0,29 & 0,41 & 0,32 \\
\hline & Densidade & & 0,86 & 0,74 & 0,83 \\
\hline 20 a 30 & Resistência & & 0,33 & 0,53 & 0,38 \\
\hline \multirow[t]{2}{*}{ Densidade } & & 0,61 & 0,77 & 0,79 & \\
\hline & & \multicolumn{4}{|c|}{ Solo Argiloso } \\
\hline Todas & Resistência & 0,67 & & & \\
\hline 0 a 10 & Resistência & & 0,57 & 0,46 & 0,42 \\
\hline Densidade & & 0,01 & 0,03 & 0,004 & \\
\hline 10 a 20 & Resistência & & 0,67 & 0,72 & 0,62 \\
\hline Densidade & & 0,72 & 0,73 & 0,64 & \\
\hline 20 a 30 & Resistência & & 0,93 & 0,71 & 0,81 \\
\hline Densidade & & 0,77 & 0,71 & 0,75 & \\
\hline
\end{tabular}

Quadro 5 - Condutividade hidráulica saturada, macroporosidade, microporosidade e volume de água disponível (entre -6 e $-1500 \mathrm{KPa}$ ) de acordo com a intensidade de tráfego na linha, em dois solos e três profundidades

Table 5 - Saturated hydraulic conductivity, macro- and microporosity and available water volume (between -6 and -1500 $\mathrm{KPa}$ ), according to the different traffic intensity in the lane, in two soils and three depths

\begin{tabular}{|c|c|c|c|c|c|}
\hline Profundidade & Linha & Condutiv. Hidrául. Saturada & Macroporosidade & Microporosidade & Água Disponível \\
\hline $\mathrm{cm}$ & & $\mathrm{cm} / \mathrm{min}$ & & $\mathrm{cm}^{3} / \mathrm{cm}^{3}$ & \\
\hline \multicolumn{6}{|c|}{ Solo Arenoso } \\
\hline \multirow[t]{3}{*}{0 a 10} & Tráfego & 0,317 & 0,381 & 0,093 & 0,063 \\
\hline & Corte & 0,347 & 0,351 & 0111 & 0,078 \\
\hline & Galhada & 0,367 & 0,381 & 0,092 & 0,064 \\
\hline \multirow[t]{3}{*}{10 a 20} & Tráfego & 0,228 & 0,301 & 0,091 & 0,059 \\
\hline & Corte & 0,292 & 0,305 & 0,104 & 0,072 \\
\hline & Galhada & 0,324 & 0,312 & 0,109 & 0,076 \\
\hline \multirow[t]{4}{*}{20 a 30} & Tráfego & 0,208 & 0,225 & 0,094 & 0,065 \\
\hline & Corte & 0,239 & 0,273 & 0,100 & 0,065 \\
\hline & Galhada & 0,247 & 0,320 & 0,122 & 0,088 \\
\hline & & & Solo & loso & \\
\hline \multirow[t]{3}{*}{0 a 10} & Tráfego & 0,042 & 0,177 & 0,335 & 0,116 \\
\hline & Corte & 0,156 & 0,214 & 0,344 & 0,125 \\
\hline & Galhada & 0,173 & 0,243 & 0,348 & 0,129 \\
\hline \multirow[t]{3}{*}{10 a 20} & Tráfego & 0,022 & 0,125 & 0,363 & 0,146 \\
\hline & Corte & 0,054 & 0,204 & 0,356 & 0,138 \\
\hline & Galhada & 0,055 & 0,214 & 0,382 & 0,157 \\
\hline \multirow[t]{3}{*}{20 a 30} & Tráfego & 0,023 & 0,162 & 0,352 & 0,132 \\
\hline & Corte & 0,131 & 0,185 & 0,381 & 0,159 \\
\hline & Galhada & 0,164 & 0,207 & 0,346 & 0,123 \\
\hline
\end{tabular}

R. Árvore, Viçosa-MG, v.29, n.3, p.383-390, 2005 


\section{CONCLUSÕES}

Com base nos dados, é possível concluir que:

a) As operações de colheita de um povoamento de eucalipto causam compactação do solo, que persiste na área de rebrota até a próxima colheita.

b) Há recuperação da estrutura do solo na camada até $10 \mathrm{~cm}$ de profundidade em área de rebrota até a próxima colheita, em ambos os solos.

c) O ponto de máxima compactação acontecem no solo argiloso na camada de 10 a $20 \mathrm{~cm}$ e no solo arenoso na camada de 20 a $30 \mathrm{~cm}$.

d) As melhores correlações com o volume de madeira da rebrota foram com densidade global, em solo arenoso; e com resistência do solo, em solo argiloso, indicando que no solo arenoso ocorre a perda da estrutura e no solo argiloso, a formação de camadas compactadas.

e) O volume de madeira, em área de rebrota, é afetado pela compactação causada pela passagem de máquinas na colheita, chegando a reduções de até dois terços nas linhas com maiores compactações do solo argiloso.

\section{REFERÊNCIAS BIBLIOGRÁFICAS}

BLACK, C.A. (Ed). Methods of soil analysis. Madison: American Society of Agronomy, 1965. v. 2. (Agronomy Monograph, 9).

\section{EMPRESA BRASILEIRA DEPESQUISA} AGROPECUÁRIA - EMBRAPA. Centro Nacional de Pesquisa de Solos. Manual de métodos de análise de solo. Rio de Janeiro, EMBRAPA/CNPS, 1997.212p. (EMBRAPA/CNPS. Documentos, 1).

FERNANDES, H.C.; VITORIA, E.L. Avaliação dos níveis de compactação de um solo florestal em relação a trafegabilidade das máquinas. Revista Árvore, v.22, p.521-526, 1998.

FROELICH, H.A.; MILES, D.W.; ROBBINS, R.W. Growth of young Pinus ponderosa and P. contorta on compacted soil in Central Washington.

Forestry Ecology Management, v.15, p.285-294, 1986.
HUANG, J.; LACEY, S.T.; RYAN, P.J. Impact of forest harvesting on the hydraulic properties of surface soil. Soil Science, v.161 p.79-86, 1996.

INCERTI, M.; CLINNICK, P.F.; WILLATT, S.T.

Changes in the physical properties of a forest soil following logging. Australian Forestry Research, v.17, p.91-98, 1987.

JAKOBSEN, B.F.; GREACEN, E.L. Compaction of sandy forest soils by forwarder operations. Soil Tillage Research, v.5, p.55 - 70, 1985.

KLUTE, A.; DIRKSEN, C. Hydraulic conductivity and diffusivity: laboratory methods. In: KLUTE, A. (Ed.). Methods of soil analysis. Physical and Mineralogical Methods. Madison, USA: American Society of Agronomy, 1986. Part I. p.687-734. (Agronomy Monograph, 9).

LACEY, S.T. et al. Soil physical property change from forest harvesting in New South Wales. West Pennant Hills: State Forest of NSW, 1994. 81 p. (Research Paper, 25).

MATTHIES, D.; WEIXLER, H.; HESS, U. Structural changes in forest soils caused by vehicle travel. AF2 Der Wald, v.50, p.12811221, 1995.

MISRA, R.K.; GIBBONS, A.K. Growth and morphology of eucalypt seedling-roots, in relation to soil strength arising from compaction. Plant Soil, v.182, p.1-11, 1996.

OLIVEIRA, L.B. Determinação de macro e microporosidade pela mesa de tensão em amostras de solo com estrutura indeformada. Pesquisa Agropecuária Brasileira, v. 3, p. 197-200, 1968.

RAB, M.A. Changes in physical properties of a soil associated with logging of E. regnans forest in Southern Australia. Forestry Ecology Management, v.70, p.215-229, 1994.

RAB, M.A. Soil physical and hydrological properties following logging and slash burning in the E. regnans forest of Southern Australia.

Forestry Ecology Management, v.84, p.159-176, 1996.

R. Árvore, Viçosa-MG, v.29, n.3, p.383-390, 2005 
SANDS, R.; GREACEN, E.L.; GERARD, C.J.

Compaction of sandy soils in radiata pine forests I. A penetrometer study. Australian Journal Soil Research, v.17, p.101-113, 1979.

SHETRON, S.G. et al. Forest soil compaction: effect of multiple passes and loadings on wheel track surface soil bulk density. Northern

Journal Applly Forestry, v.5, p.120-123, 1988.

THEODOROU, C.; CAMERON, J.N.; BOWEN, G.D. Growth of roots of different Pinus radiata genotypes in soil at different strength and aeration. Australian Forestry, v.54, p.52-59, 1991.
VETTORI, L. Métodos de análises de solos. Rio de Janeiro: Equipe de Pedologia e Fertilidade do Solo, 1969. 24 p. (Boletim Técnico, 7).

VOORHEES, W.B. et al. Corn growth and yield as affected by surface and subsoil compaction. Agronomy Journal, v.81, p.294 - 303, 1989.

WARKOTSCH, P.W.; van HUYSSTEEN, L.; OLSEN, G.J. Identification and quantification of soil compaction due to various harvesting methods - a case study. South African, Forestry Journal, v.170, p.7 -15, 1994. 